\title{
EXISTENCE AND UNIQUENESS OF SOLUTIONS OF CERTAIN SYSTEMS OF ALGEBRAIC EQUATIONS WITH OFF DIAGONAL NONLINEARITY
}

\author{
JÜRGEN FUHRMANN
}

\begin{abstract}
Using inverse positivity properties and Brouwer's fixed point theorem, we derive existence and uniqueness results for certain nonlinear systems of equations with off diagonal nonlinearity. The type of systems considered arises from stable finite volume discretizations of viscous nonlinear conservation laws and has a wide range of applications.
\end{abstract}

\section{INTRODUCTION}

Existence and uniqueness results for nonlinear systems of equations in $\mathbb{R}^{n}$ with arbitrary right hand sides can be given only under structural restrictions to the system. One well understood class of systems in this respect has the structure

$$
B(u)+A u=f
$$

where $A$ is an M-matrix and $B: \mathbb{R}^{n} \rightarrow \mathbb{R}^{n}$ is an isotone diagonal mapping [OR70]. This type of systems arises e.g. from the nonlinear Poisson equation in semiconductor device simulation when discretized with finite volume methods [Sel84, GLTZ89]. More general nonlinear systems of equations one can find when numerically treating nonlinear heat transfer [FH97] or porous media flow problems [Ric31, Fuh97]. When discretized following certain rules, in each time step of the solution algorithm for these problems we have to solve a system of the form

$$
B(u)+A(u)+Q(u)=B\left(u_{0}\right)
$$

with $B$ being an isotone diagonal operator, $Q(u)$ limited and $A(u)$ being an operator with off-diagnal nonlinearities and certain structural properties.

In this paper, we prove existence and uniqueness results for (1.2), also for cases with degenerating $B$ or $A$. The basic idea of the proof is the usage of estimates of inverse matrix norms from the Perron-Frobenius theory in Brouwer's fixed point theorem.

The paper is organized as follows. In section 2 we prove a basic existence result using Brouwer's fixed point theorem. Section 3 contains existence results based on $L^{\infty}$-estimates and weakly row-wise diagonal dominance. Section 4 is devoted to existence and uniqueness considerations in the case of $L^{1}$-estimates and weakly column-wise diagonal dominance. As the considerations in this paper are motivated by finite volume discretizations of initial boundary value problems for nonlinear parabolic partial differential equations, we introduce algebraically the notation of a "Dirichlet"-problem and prove our results for this case as well. To illustrate what we have in mind, section 5 contains an example of a finite difference

Date: August 14, 1998.

1991 Mathematics Subject Classification. 15A48, 65E99, 65H10, 65M12.

Key words and phrases. systems of algebraic equations, positive matrices, viscous conservation laws, finite volume methods, nonlinear parabolic PDEs.

Email.fuhrmann@wias-berlin.de.

Address. Weierstraß Institute for Applied Analysis and Stochastics, Mohrenstr. 39, 10117 Berlin, Germany.

WIAS Preprint no. 425. 
scheme for a nonlinear parabolic problem in an one-dimensional domain. Finally, appendix A explains our notations, and for the convenience of the reader, collects some well known definitions and implications of the Perron-Frobenius theory. Appendix $B$ contains the formulation of a mean value theorem we often use.

\section{A BASIC EXISTENCE THEOREM}

The following theorem is the basic building block of the theory developed in this paper.

2.1. Theorem. Let $K \in C\left(\mathbb{R}^{n}, \mathbb{M}(n)\right)$ and $Q \in C\left(\mathbb{R}^{n}, \mathbb{R}^{n}\right)$. For any $u \in \mathbb{R}^{n}$ assume either

$$
K(u) \in \mathbb{Z}_{r}^{+}(n) \text { and }\|Q(u)\|_{\infty} \leq Q_{\infty}^{+}
$$

or

$$
K(u) \in \mathbb{Z}_{c}^{+}(n) \text { and }\|Q(u)\|_{1} \leq Q_{1}^{+} \text {. }
$$

Then the equation

$$
u+K(u) u=Q(u)
$$

has a solution $\hat{u}$ with either

$$
\|\hat{u}\|_{\infty} \leq Q_{\infty}^{+}
$$

or

$$
\|\hat{u}\|_{1} \leq Q_{1}^{+},
$$

respectively.

Proof. The solution $\hat{u}$ would be a fixed point of the mapping

$$
L(u)=(I+K(u))^{-1} Q(u) .
$$

For $\xi \in\{1, \infty\}$ theorems A.7,A.9 give us

$$
\left\|(I+K(u))^{-1}\right\|_{\xi} \leq 1,
$$

hence

$$
\|L(u)\|_{\xi} \leq Q_{\xi}^{+} .
$$

The entries of $(I+K(u))^{-1}$ are rational functions of the entries of $I+K(u)$. Inverse positivity bounds them below by zero, and the norm estimate together with the explicit norm representation (A.4) bounds them from above. Hence for $K \in \mathbb{Z}_{r}^{+}(n)$ respectively $K \in \mathbb{Z}_{c}^{+}(n)$ these rational functions cannot have poles and need to be continuous. Thus the mapping $L$ is continuous as well. As the balls $\left\{u \in \mathbb{R}^{n}|\quad| \mid u \|_{\xi}<Q_{\xi}^{+}\right\}$are convex, we can apply Brouwer's fixed point theorem which ensures the existence of the desired fixed point which at the same time admits the estimates (2.4a) and (2.4b), respectively.

\section{NONNEGATIVE ROW SUMS AND $L^{\infty}$ ESTIMATES}

In this section, we generalize theorem 2.1 for the case of nonnegative row sums and $L^{\infty}$ - estimates. The nonlinear operators we have in mind here come from discretizations of nonlinear diffusion problems by the finite volume method or by the finite element method with quadrature rules. An introductory example one finds in section 5 .

Having in mind the application of our theory in the framework of numerical methods for the solution of initial boundary value problems for partial differential equations, we introduce here the notation of a Dirichlet problem. For simplicity, we do this strictly algebraically, as it can be done in numerical codes as well. 
In this case, a Dirichlet problem is posed just by fixing the values of a set $D$ of unknowns and regarding only the equations corresponding to the complementary set of unknowns $I=\{1 \ldots n\} \backslash D$.

So, assume a partition of our index set $\{1 \ldots n\}=D \cup I$ into the sets of "Dirichlet nodes" and "interior nodes". The corresponding splitting $\mathbb{R}^{n}=\mathbb{R}_{D} \oplus \mathbb{R}_{I}$ for any matrix $A \in \mathbb{M}(n)$ after an obvious reordering of unknowns induces a splitting

$$
A=\left(\begin{array}{cc}
A_{D D} & A_{D I} \\
A_{I D} & A_{I I}
\end{array}\right) \text {. }
$$

For any vector $u \in \mathbb{R}^{n}$ we introduce the coordinate projections $u_{D} \in \mathbb{R}_{D}$ and $u_{I} \in$ $\mathbb{R}_{I}$. In this case we can write

$$
u=\left(\begin{array}{c}
u_{D} \\
u_{I}
\end{array}\right) .
$$

The restriction of a nonlinear operator $B: \mathbb{R}^{n} \rightarrow \mathbb{R}^{n}$ to $I$ we denote by $B_{I}: \mathbb{R} \rightarrow \mathbb{R}_{I}$.

For simplicity, we introduce the follwing notation which is similar to the one used for partial differential equations. Please note that everywhere we regard Dirichlet problems, we allow $D$ to be the empty set.

3.2. Definition. Let $B: \mathbb{R}^{n} \rightarrow \mathbb{R}^{n}$ be a diagonal operator, $Q: \mathbb{R}^{n} \rightarrow \mathbb{R}^{n}$ be arbitrary and $K: \mathbb{R}^{n} \rightarrow \mathbb{M}(n)$ be a matrix valued function. Let $u_{0} \in \mathbb{R}^{n}$ and $u_{d} \in \mathbb{R}_{D}$ be given vectors. We say that $u$ is a solution of the Dirichlet problem

$$
\begin{cases}B(u)+K(u) u & =Q(u)+B\left(u_{0}\right) \\ \left.u\right|_{D} & =u_{d}\end{cases}
$$

if

$$
u_{D}=u_{d}
$$

and

$$
B_{I}(u)+K_{I I}(u)=Q_{I}(u)+B_{I}\left(u_{0}\right)-K_{I D}(u) u_{d}
$$

3.5. Theorem. Let $K \in C\left(\mathbb{R}^{n}, \mathbb{M}(n)\right)$ and $Q \in C\left(\mathbb{R}^{n}, \mathbb{R}^{n}\right)$. For any $u \in \mathbb{R}^{n}$ assume

$$
K(u) \in \mathbb{Z}_{r}^{+}(n)
$$

and

$$
\|Q(u)\|_{\infty} \leq Q^{+}
$$

Further, let $B \in C^{1}\left(\mathbb{R}^{n}, \mathbb{R}^{n}\right)$ be a diagonal operator with

$$
\left\|B^{\prime}(u)\right\|_{\infty} \geq B^{-}>0 .
$$

Then for any $u_{0} \in \mathbb{R}^{n}$, for any (possibly empty) subset $D \subset\{1 \ldots n\}$ and any $u_{d} \in \mathbb{R}_{D}$, the Dirichlet problem (3.3) has a solution $\hat{u}$ with

$$
\left\|\hat{u}_{I}\right\|_{\infty} \leq \frac{Q^{+}}{B^{-}}+\left\|u_{0, I}\right\|_{\infty}+\left\|u_{d}\right\|_{\infty}
$$

assuming $\left\|u_{d}\right\|_{\infty}=0$ for $D=\emptyset$.

Proof. Let $\tilde{B}_{I}\left(u, u_{0}\right)$ be the diagonal matrix defined by the mean value theorem B.1. It allows us to rewrite equation (3.4b) by

$$
\tilde{B}_{I}\left(u, u_{0}\right) u_{I}+K_{I I}(u)=Q_{I}(u)+\tilde{B}_{I}\left(u, u_{0}\right) u_{0, I}-K_{I D}(u) u_{d} .
$$

By the identity (B.3), any solution of (3.8a) is a solution of (3.4b).

Further, $K_{I I}(u) \in \mathbb{Z}_{r}^{+}(I)$, and we have a splitting

$$
K_{I I}(u)=K^{0}(u)+K^{D}(u)+K^{M}(u)
$$


with $K^{0}(u) \in \mathbb{Z}_{r}^{0}(I), K^{D}(u) \in \mathbb{D}^{+}(I)$ being the negative row sum of $K_{I D}(u)$ and $K^{M}(u) \in \mathbb{D}^{+}(I)$ being some nonnegative diagonal matrix.

Let

$$
M(u)=\tilde{B}_{I}\left(u, u_{0}\right)+K^{D}(u)+K^{M}(u) .
$$

We have $M(u) \in \mathbb{D}^{++}(I)$ and can rewrite equation (3.8a) by

$$
M(u) u_{I}+K^{0}(u) u_{I}=Q_{I}(u)+\tilde{B}_{I}\left(u, u_{0}\right) u_{0, I}-K_{I D}(u) u_{d}
$$

and

$$
u_{I}+M(u)^{-1} K^{0}(u) u_{I}=M(u)^{-1}\left(Q_{I}(u)+\tilde{B}_{I}\left(u, u_{0}\right) u_{0, I}-K_{I D}(u) u_{d}\right) .
$$

By theorem A.7, $M(u)^{-1} K^{0}(u) \in \mathbb{Z}_{r}^{+}(I)$. Further, it continuously depends on $u$. We can estimate

$$
\begin{aligned}
\left\|M(u)^{-1} Q_{I}(u)\right\|_{\infty} & \leq \frac{Q^{+}}{B^{-}} \\
\left\|M(u)^{-1} B_{I}\left(u, u_{0}\right)\right\|_{\infty} & \leq 1 \\
\left\|M(u)^{-1} K_{I D}(u)\right\|_{\infty} & \leq 1
\end{aligned}
$$

because the row sums of $M(u)^{-1} K_{I D}(u)$ are less then one by construction.

Hence, by theorem 2.1, equation (3.8c) has a solution which can be estimated by (3.7). It is a solution of (3.4b) as well, and by definition, it is a solution of the Dirichlet problem (3.3).

If $D=\emptyset$, we can everywhere omit $u_{d}, K_{I D}$ and $K^{D}$, thus obtaining the result also for this case.

3.9. Remark. Please note, that we did not demand any non-degeneracy condition for $K(u)$. This allows to apply our result also in the case of degenerating diffusion equations, where rows of $K(u)$ can become zero.

Another case of degeneracy which occurs in saturated/unsaturated flow calculations [Ric31] is covered by the next theorem.

3.10. Theorem. Let $K \in C\left(\mathbb{R}^{n}, \mathbb{M}(n)\right)$. For any $u \in \mathbb{R}^{n}$ assume

$$
K(u) \in \mathbb{Z}_{r}^{+}(n)
$$

Further, let $B \in C^{1}\left(\mathbb{R}^{n}, \mathbb{R}^{n}\right)$ be a diagonal operator with

$$
B^{\prime}(u) \geq B^{-} \geq 0 \text {. }
$$

Then for any $u_{0} \in \mathbb{R}^{n}$, for any (possibly empty) subset $D$ and any $u_{d} \in \mathbb{R}_{D}$, the Dirichlet problem

$$
\begin{cases}B(u)+K(u) u & =B\left(u_{0}\right) \\ \left.u\right|_{D} & =u_{d}\end{cases}
$$

has a solution $\hat{u}$ with

$$
\left\|\hat{u}_{I}\right\|_{\infty} \leq\left\|u_{0, I}\right\|_{\infty}+\left\|u_{d}\right\|_{\infty}
$$

assuming $\left\|u_{d}\right\|_{\infty}$ for $D=\emptyset$.

Proof. For $\varepsilon>0$ Let $B_{\varepsilon}(u)=B(u)+\varepsilon u$. Then after theorem 3.5, the Dirichlet problem

$$
\begin{cases}B_{\varepsilon}(u)+K(u) u & =B_{\varepsilon}\left(u_{0}\right) \\ \left.u\right|_{D} & =u_{d}\end{cases}
$$

has a solution $u_{\varepsilon}$ which can be estimated by

$$
\left\|u_{\varepsilon, I}\right\|_{\infty} \leq\left\|u_{0, I}\right\|_{\infty}+\left\|u_{d}\right\|_{\infty}
$$


Hence we can find an element $\hat{u}_{I}$ admitting the estimate (3.13) and a sequence $\varepsilon_{n}$ with

$$
\begin{aligned}
\lim _{n \rightarrow \infty} \varepsilon_{n} & =0, \\
\lim _{n \rightarrow \infty} u_{\varepsilon_{n}} & =\hat{u} .
\end{aligned}
$$

As all operators involved continuously depend on $u, \hat{u}=\left(\begin{array}{l}u_{d} \\ \hat{u}_{I}\end{array}\right)$ necessarily is a solution of (3.12).

3.14. Remark. Unfortunately, using the type of estimates of this section, we are not able to prove uniqueness. At the other hand, in the case of theorem 3.10, for both degenerating $B$ and $K$, we of course have to expect multiple solutions. Further one can expect that already in the case of linear $B$ and $K$ that for certain $Q$, bifurcations happen.

\section{NONNEGATIVE COLUMN SUMS AND $L^{1}$ ESTIMATES}

In this section, we apply theorem 2.1 to the case of nonnegative column sums and $L^{1}$ - estimates. The nonlinear operators corresponding to this case come from stable finite volume discretizations of nonlinear convection diffusion problems. Under certain conditions, the example from section 5 is valid for this case, as well.

4.1. Theorem. Let $A \in C\left(\mathbb{R}^{n}, \mathbb{R}^{n}\right)$ have a Gâteaux derivative $A^{\prime}(u) \in \mathbb{Z}_{c}^{+}(n)$ continuously depending on $u$. Further, let $B \in C^{1}\left(\mathbb{R}^{n}, \mathbb{R}^{n}\right)$ be an isotone diagonal homeomorphism. Let $Q \in C\left(\mathbb{R}^{n}, \mathbb{R}^{n}\right)$ be bounded with $\|Q(u)\|_{1} \leq Q^{+}$. Then for $u_{0} \in \mathbb{R}^{n}$, for any (possibly empty) subset $D \subset\{1 \ldots n\}$, and $u_{d} \subset \mathbb{R}_{D}$, the Dirichlet problem

$$
\left\{\begin{array}{l}
B(u)+A(u)=Q(u)+B\left(u_{0}\right) \\
\left.u\right|_{D}=u_{d}
\end{array}\right.
$$

has a solution $\hat{u}$ which in the case $D=\emptyset$ admits the estimate

$$
\|B(u)\|_{1} \leq\left\|B\left(u_{0}\right)\right\|_{1}+Q^{+}+\left\|A\left(B^{-1}(0)\right)\right\|_{1} .
$$

Proof. First, regard the case $D=\emptyset$. As $B$ is an homeomorphism, we can perform a variable substitution $v=B(u)$.

Let $A_{B}(v)=A\left(B^{-1}(v)\right)$. We have the identity $A_{B}^{\prime}(v)=A^{\prime}\left(B^{-1}(v)\right)\left(B^{-1}\right)^{\prime}(v)$, thus because of theorem A.9, the isotonicity and the continuity of $B$ and hence of its inverse, $A_{B}$ fulfills the conditions of the theorem. Thus we restrict ourselves to the problem

$$
v+A_{B}(v)=Q_{B}(v)+v_{0}
$$

whith $Q_{B}(v)=Q\left(B^{-1}(v)\right)$ and $v_{0}=B\left(u_{0}\right)$.

Let $A_{B 0}(v)=A_{B}(v)-A_{B}(0)$ and $Q_{B 0}(v)=Q_{B}(v)-A_{B}(0)$. We get the equivalent problem

$$
v+A_{B 0}(v)=Q_{B 0}(v)+v_{0}
$$

where $A_{B 0}$ besides of the condition of the theorem fulfills $A_{B 0}(0)=0$ and $Q_{B 0}$ is limited by

$$
\left\|Q_{B 0}(v)\right\|_{1} \leq Q_{B 0}^{+}=Q^{+}+\left\|A_{B}(0)\right\|_{1}=Q^{+}+\left\|A\left(B^{-1}(0)\right)\right\|_{1}
$$

Let $\tilde{A}_{B 0}(v, w) \in \mathbb{Z}_{c}^{+}(n)$ be defined as in theorem B.1. Then we can rewrite equation $(4.4 b)$ to

$$
v+\tilde{A}_{B 0}(v, 0) v=Q_{B 0}(v)+v_{0}
$$


which after theorem 2.1 has a solution $\hat{v}$ with the estimate

$$
\|\hat{v}\|_{1} \leq Q_{B 0}^{+}+\left\|v_{0}\right\|_{1} .
$$

It is a solution of $(4.4 \mathrm{~b})$, too and consequently, $\hat{u}=B^{-1}(\hat{v})$ is a solution of (4.2) admitting the estimate (4.3).

For the Dirichlet problem, we regard

$$
B_{I}(u)+A_{I}(u)=Q_{I}(u)+B_{I}\left(u_{0}\right)
$$

We have a splitting

$$
A^{\prime}(u)=\left(\begin{array}{cc}
A_{D D} & A_{D I} \\
A_{I D} & A_{I I}
\end{array}\right)
$$

As $A^{\prime}(u) \in \mathbb{Z}_{c}^{+}(n), A_{I I} \in \mathbb{Z}_{c}^{+}(I)$, thus (4.4e) fulfills the conditions of the first part of the proof.

4.5. Remark. Again, degeneracy of the "main part" $A$ is allowed. But unlike in section 3 , the existence proof for degenerating $B$ does not work here.

4.6. Theorem. Assume that the conditions of theorem 4.3 are fulfilled, that $Q=0$ and that for any $u \in \mathbb{R}^{n}, B^{\prime}(u) \in \mathbb{D}^{++}(n)$. Then the solution of (4.2) is unique.

Proof. For $u, v$ being two solutions of (4.2) after theorem B.1 we can write

$$
\tilde{B}(u, v)(u-v)+\tilde{A}(u, v)(u-v)=0 .
$$

By theorem A.9, $\tilde{B}(u, v)+\tilde{A}(u, v)$ is an M-Matrix, thus necessarily, $u=v$.

\section{EXAMPLE: NONLINEAR DIFFUSION IN INHOMOGENEOUS DOMAINS}

To motivate our considerations, we give a simple example. In the fields of nonlinear heat transfer and of porous media flow simulation, we have to solve numerically initial boundary value problems of the form

$$
\partial_{t} b(x, u(x, t))-\nabla \cdot(d(x, u(x, t)) \nabla u(x, t))=0 .
$$

with $b$ monotonically increasing and continuously differentiable in $u$ and $d$ continuous and nonnegative. For simplicity, we assume here an one-dimensional domain $\Omega=(0,2)$ with two subdomains $\Omega_{1}=(0,1)$ and $\Omega_{2}=(1,2)$ with homogeneous Neumann boundary conditions, and an initial value $u_{0}$. Assume that $b(x, u)=b^{m}(u)$ and $d(x, u)=d^{m}(u)$ on $\Omega_{m}$, respectively.

If we introduce an implicit Euler time discretization with timestep $\tau$, in the $\nu$ th timestep we have to solve a problem of the type

$$
b\left(x, u_{\nu}\right)-\tau \nabla \cdot\left(d\left(x, u_{\nu}\right) \nabla u_{\nu}\right)=b\left(x, u_{\nu-1}\right)
$$

To be able to do this, we introduce the following finite difference scheme:

Let $N=2 n, h=\frac{1}{n}$ and for $i=0 \ldots 2 n$, let $x_{i}=i h$, and $u_{i} \approx u\left(x_{i}\right)$. Let

$$
B(u)_{i}=h \begin{cases}\frac{1}{2} b^{1}\left(u_{i}\right), & i=0 \\ b^{1}\left(u_{i}\right), & 0<i<n \\ \frac{1}{2}\left(b^{1}\left(u_{i}\right)+b^{2}\left(u_{i}\right)\right), & i=n \\ b^{2}\left(u_{i}\right), & n<i<2 n \\ \frac{1}{2} b^{2}\left(u_{i}\right), & i=2 n\end{cases}
$$


and

$$
A(u)_{i}=\frac{\tau}{h}\left\{\begin{aligned}
g^{1}\left(u_{i}, u_{i+1}\right), & i=0 \\
g^{1}\left(u_{i}, u_{i-1}\right)+g^{1}\left(u_{i}, u_{i+1}\right), & 0<i<n \\
g^{1}\left(u_{i}, u_{i-1}\right)+g^{2}\left(u_{i}, u_{i+1}\right), & i=n \\
g^{2}\left(u_{i}, u_{i-1}\right)+g^{2}\left(u_{i}, u_{i+1}\right), & n<i<2 n \\
g^{2}\left(u_{i}, u_{i-1}\right), & i=2 n
\end{aligned}\right.
$$

Then under certain conditions on $g^{1,2}$, we can regard

$$
B\left(u_{\nu}\right)+A\left(u_{\nu}\right)=B\left(u_{\nu-1}\right)
$$

as an approximation to (5.2).

A common way to discretize the nonlinear diffusion term is the usage of the arithmetical mean of the solutions of the neigboring nodes:

$$
g^{m}(\xi, \eta)=d^{m}\left(\frac{\xi+\eta}{2}\right)(\xi-\eta)
$$

If $b^{m \prime}>b^{-} \geq 0$, for $A$ and $B$, the conditions of theorem 3.10 are fulfilled. Thus for arbitrarily large timesteps, the time step problems are solvable, and, furthermore, the estimate (3.13) yields the $L^{\infty}$ stability estimate

$$
\left\|u_{\nu}\right\|_{\infty} \leq\left\|u_{\nu-1}\right\|_{\infty} \text {. }
$$

Please note, that $d$ can become zero, thus allowing to consider degenerate diffusion effects. However, $A$ is not of isotone flux type, and even in the case of strictly montone $b$ we are not able to prove uniqueness. The practical experience however does not indicate that multiple solutions ar a problem in this case [Fuh97, FH97].

The natural question if there exists as scheme which allows for uniqueness is answered by the following variant: Let $d^{m}=D^{m \prime}$ and define

$$
g^{m}(\xi, \eta)=D^{m}(\xi)-D^{m}(\eta)
$$

The mean value theorem says that there exists a $\lambda \in[\xi, \eta]$ such that

$$
g^{m}(\xi, \eta)=d^{m}(\lambda)(\xi-\eta)
$$

thus still being consistent to the diffusion type operator. The so defined operator $A(u)$ now fulfills the conditions of theorem 4.1. Defining

$$
k^{m}(\xi, \eta)= \begin{cases}\frac{D^{m}(\xi)-D^{m}(\eta)}{\xi-\eta}, & \xi \neq \eta \\ d^{m}(\xi), & \xi=\eta\end{cases}
$$

for $D \in C^{1}(\mathbb{R}, \mathbb{R})$ allows to rewite $A(u)=K(u) u$ with $K$ continuously depending on $u$ :

$$
K(u)_{i j}=\frac{\tau}{h} \begin{cases}k^{1}\left(u_{i}, u_{j}\right), & 0<=i<n, j=i+1 \\ k^{1}\left(u_{i}, u_{j}\right), & 0<i<=n, j=i-1 \\ k^{2}\left(u_{i}, u_{j}\right), & n<=i<2 n, j=i+1 \\ k^{2}\left(u_{i}, u_{j}\right), & n<i<=2 n, j=i-1 \\ 0, & \text { else }\end{cases}
$$

Thus, the conditions of both theorems 3.10 and 4.6 are fulfilled, and the solutions of the timestep problems are unique.

We remark that in the case of homogeneous materials, i.e. $b^{1}=b^{2}$ and $d^{1}=d^{2}$ we could use the variable transformation $v=D(u)$. In this case, the results for the case of diagonal nonlinearity would be sufficient.

We further remark, that a generalization of these results to higher space dimensions, unstructured meshes and more general conservation laws one finds in the forthcoming paper [FL98]. 


\section{APPENDIX A. LINEAR ALgEBRA}

A good reference for the following results still is [Var62]. Let $\mathbb{M}(n)$ be the set of all real $n \times n$-matrices.

A.1. Definition. For $u=\left(u_{i}\right)_{i=1 \ldots n} \in \mathbb{R}^{n}$, let

$$
\begin{aligned}
\|u\|_{1} & =\sum_{i=1}^{n}\left|u_{i}\right| \\
\|u\|_{\infty} & =\max _{i=1}^{n}\left|u_{i}\right|
\end{aligned}
$$

A.3. Remark. Let $A \in \mathbb{M}(n)$. Then

$$
\begin{aligned}
& \|A\|_{1}=\sup _{0 \neq u \in \mathbb{R}^{n}} \frac{\|A u\|_{1}}{\|u\|_{1}}=\max _{j=1}^{n} \sum_{i=1}^{n}\left|a_{i j}\right|=\left\|A^{T}\right\|_{\infty} \\
& \|A\|_{\infty}=\sup _{0 \neq u \in \mathbb{R}^{n}} \frac{\|A u\|_{\infty}}{\|u\|_{\infty}}=\max _{i=1}^{n} \sum_{j=1}^{n}\left|a_{i j}\right|=\left\|A^{T}\right\|_{1}
\end{aligned}
$$

Further, we introduce some notations which are not usual, but we think they are useful in the context of this paper. We note that often there isn't paid attention to the difference between row-wise and column-wise diagonal dominance.

A.5. Definition. Define the following subsets of $\mathbb{M}(n)$

\begin{tabular}{|l|l|}
\hline $\mathbb{Z}(n) \subset \mathbb{M}(n)$ & $\begin{array}{l}\text { nonnegative main diagonal } \\
\text { and nonpositive off-diagonal } \\
\text { elements (often denoted as } \\
\left.Z^{n \times n}\right)\end{array}$ \\
\hline $\mathbb{Z}_{c}^{+}(n) \subset \mathbb{Z}^{(n)}$ & $\begin{array}{l}\text { columnwise weakly diagonally } \\
\text { dominant }\end{array}$ \\
\hline $\mathbb{Z}_{c}^{++}(n) \subset \mathbb{Z}_{c}^{+}(n)$ & $\begin{array}{l}\text { columnwise strictly diagonally } \\
\text { dominant }\end{array}$ \\
\hline $\mathbb{Z}_{c}^{0}(n) \subset \mathbb{Z}_{c}^{+}(n)$ & column sum zero \\
\hline $\mathbb{Z}_{r}^{+}(n) \subset \mathbb{Z}^{(n)}$ & $\begin{array}{l}\text { row wise weakly diagonally } \\
\text { dominant }\end{array}$ \\
\hline $\mathbb{Z}_{r}^{0}(n) \subset \mathbb{Z}_{r}^{+}(n)$ & row sum zero \\
\hline $\mathbb{Z}_{r}^{++}(n) \subset \mathbb{Z}_{r}(n)$ & $\begin{array}{l}\text { row wise strictly diagonally } \\
\text { dominant }\end{array}$ \\
\hline $\mathbb{D}_{(n) \subset \mathbb{M}^{+}(n)}$ & diagonal \\
\hline $\mathbb{D}^{+}(n) \subset \mathbb{D}^{(}(n) \cap \mathbb{Z}_{r}^{+}(n) \cap \mathbb{Z}_{c}^{+}(n)$ & nonnegative diagonal \\
\hline $\mathbb{D}^{++}(n) \subset \mathbb{D}^{+}(n) \cap \mathbb{Z}_{r}^{++}(n) \cap \mathbb{Z}_{c}^{++}(n)$ & positive diagonal \\
\hline
\end{tabular}

For a subset $X \subset\{1 \ldots n\}$ we write $\mathbb{M}(X), \mathbb{Z}(X)$ etc. to denote the sets of matrices corresponding to this subset.

$A=\left(a_{i j}\right)_{i, j=1 \ldots n} \in \mathbb{M}(n)$ is called positive $(A>0)$ if $a_{i j}>0, i, j=1 \ldots n$ and nonnegative $(A \geq 0)$ if $a_{i j} \geq 0, i, j=1 \ldots n$

A.6. Definition. A Matrix $A \in \mathbb{Z}(n)$ is called (nonsingular) $M$-matrix if $A^{-1} \geq 0$.

A.7. Theorem. Let $A \in \mathbb{Z}_{r}^{+}(n), D \in \mathbb{D}^{++}(n)$. Then

(i). $D A \in \mathbb{Z}_{r}^{+}(n)$

(ii). $A+D \in \mathbb{Z}_{r}^{++}(n)$ is an M-matrix

(iii). $\left\|(I+A)^{-1}\right\|_{\infty} \leq 1$

Proof. The first part is obvious, for the second, see [Axe94], lemma 6.2. For the third part, let first $A \in \mathbb{Z}_{r}^{0}(n)$. 
Assume that $\left\|(I+A)^{-1}\right\|>1$. We know that $(I+A)^{-1}$ has positive entries. Then for $\alpha_{i j}$ being the entries of $(I+A)^{-1}$,

$$
\max _{i=1}^{n} \sum_{j=1}^{n} \alpha_{i j}>1 .
$$

Let $k$ be a row where the maximum is reached. Let $e=(1 \ldots 1)^{T}$. Then for $v=$ $(I+A)^{-1} e$ we have that $v>0, v_{k}>1$ and $v_{k} \geq v_{j}$ for all $j \neq k$. The $k$ th equation of $e=(I+A) v$ then looks like

$$
\begin{aligned}
1 & =v_{k}+v_{k} \sum_{j \neq k}\left|a_{k j}\right|-\sum_{j \neq k}\left|a_{k j}\right| v_{j} \\
& \geq v_{k}+v_{k} \sum_{j \neq k}\left|a_{k j}\right|-\sum_{j \neq k}\left|a_{k j}\right| v_{k} \\
& =v_{k} \\
& >1
\end{aligned}
$$

This contradiction enforces $\left\|(I+A)^{-1}\right\|_{\infty} \leq 1$.

Let now $A \in \mathbb{Z}_{r}^{+}(n)$. The we can write $A=D+A_{0}$ with $D \in \mathbb{D}^{+}(n)$ and $A \in$ $\mathbb{Z}_{c}^{0}(n)$. We have

$$
\begin{aligned}
I+A & =I+D+A_{0} \\
& =(I+D)(I+D)^{-1}\left(I+D+A_{0}\right) \\
& =(I+D)\left(I+A_{D 0}\right)
\end{aligned}
$$

with $A_{D 0}=(I+D)^{-1} A_{0} \in \mathbb{Z}_{c}^{0}(n)$. Thus

$$
\begin{aligned}
\left\|(I+A)^{-1}\right\|_{\infty} & =\left\|\left(I+A_{D 0}\right)^{-1}(I+D)^{-1}\right\|_{\infty} \\
& \leq\left\|(I+D)^{-1}\right\|_{\infty} \\
& \leq 1,
\end{aligned}
$$

because all main diagonal entries of $I+D$ are greater or equal to 1 .

A.8. Remark. The third part of the theorem comes from the M-criterion of [GR92, RST96], both refer to [Boh81].

A.9. Theorem. Let $A \in \mathbb{Z}_{c}^{+}(n), D \in \mathbb{D}^{++}(n)$. Then

(i). $A D \in \mathbb{Z}_{c}^{+}(n)$

(ii). $A+D \in \mathbb{Z}_{c}^{++}(n)$ is an M-matrix

(iii). $\left\|(I+A)^{-1}\right\|_{1} \leq 1$

Proof. Apply theorem (A.7) to $A^{T}$.

\section{APPENDiX B. A MEAN VALUE THEOREM}

Here, we state a mean value theorem which several times is applied in this paper. We are indebted to L. Recke for the hint to use this type of mean value problem within our considerations.

B.1. Theorem. Let $A: \mathbb{R}^{n} \rightarrow \mathbb{R}^{n}$ be Gâteaux differentiable for any $u \in \mathbb{R}^{n}$ with the Gâteaux derivative $A^{\prime}(u)$. Assume $A^{\prime} \in C\left(\mathbb{R}^{n}, \mathbb{M}(n)\right)$. Then for any $u, v \in \mathbb{R}^{n}$, the operator $\tilde{A}(u, v)$ defined by

$$
\tilde{A}(u, v)=\int_{0}^{1} A^{\prime}(v+\theta(u-v)) d \theta
$$


satisfies $\tilde{A} \in C\left(\mathbb{R}^{n} \times \mathbb{R}^{n}, \mathbb{M}(n)\right)$ and

$$
A(u)-A(v)=\tilde{A}(u, v)(u-v) .
$$

If $A^{\prime}(u)$ independently of $u$ belongs to any of the subsets of $M(n)$ from definiton A.5, then $\tilde{A}(u, v)$ does.

Proof. The existence of $\tilde{A}$ satisfying (B.3) is proven in [OR70]. The nonzero pattern, the sign pattern and the row/column sum conditions are straightforward.

\section{REFERENCES}

[Axe94] O. AxELSSON, Iterative solution methods, Cambridge Unversity Press, Cambridge, 1994.

[Boh81] E. BOHL, Finite Modelle gewöhnlicher Randwertaufgaben, Teubner, Stuttgart, 1981.

[FH97] J. FUHRMANN AND D. HÖMBERG, Numerical simulation of surface heat treatments, preprint 375, Weierstraß-Institut für Angewandte Analysis und Stochastik, 1997. submitted.

[FL98] J. FUHRMANN AND H. LANGMACH, Stability of solutions of time-implicit finite volume schemes for viscous nonlinear conservation laws. WIAS Preprint, 1998. in preparation.

[Fuh97] J. FUHRMANN, On numerical solution methods for nonlinear parabolic problems, in Modeling and Computation in Environmental Sciences. Proceedings of the First GAMM-Seminar at ICA Stuttgart, October 12-13,1995, R. Helmig, W. Jäger, W. Kinzelbach, P. Knabner, and G. Wittum, eds., vol. 59 of Notes on Numerical Fluid Mechanics, Braunschweig, 1997, Vieweg, 170180.

[GLTZ89] H. GAJEWSKI, H. LANGMACH, G. TELSCHOW, AND K. ZACHARIAS, Der 2DBauelementesimulator TOSCA - Handbuch, technical documentation, Karl-Weierstraß-Institut für Mathematik, Berlin, 1989. unpubl.

[GR92] C. GROSSMANn AND H.-G. RoOS, Numerik partieller Differentialgleichungen, Teubner Studienbücher Mathematik, Teubner, Stuttgart, 1992.

[OR70] J. M. ORTEGA AND W. C. RHEINBOLDT, Iterative solution of nonlinear equations in several variables, Academic Press, New York, 1970.

[Ric31] L. A. RICHARDS, Capillary conduction of liquids through porous mediums, Physics, 1 (1931), 318333.

[RST96] H.-G. RoOs, M. STYNES, AND L. TOBISKA, Numerical Methods for Singularly Perturbed Differential Equations, no. 24 in Springer Series in Computational Mathematics, Springer, 1996.

[Sel84] S. SELbERHERR, Analysis and simulation of semiconductor devices, Computational Microelectronics, Springer Verlag, Wien, 1984.

[Var62] R. S. VARGA, Matrix iterative analysis, Prentice-Hall, Englewood Cliffs, NJ, 1962. 